\title{
LOCAL MARTINGALES AND FILTRATION SHRINKAGE
}

\author{
HANS FÖLlmer ${ }^{1}$ AND Philip ProtTer ${ }^{2}$
}

\begin{abstract}
A general theory is developed for the projection of martingale related processes onto smaller filtrations, to which they are not even adapted. Martingales, supermartingales, and semimartingales retain their nature, but the case of local martingales is more delicate, as illustrated by an explicit case study for the inverse Bessel process. This has implications for the concept of No Free Lunch with Vanishing Risk, in Finance.
\end{abstract}

Mathematics Subject Classification. 60G44, 60H05, 60J65, 60J55.

Received December 1, 2009.

\section{INTRODUCTION}

It is a classic result, known as Stricker's theorem, that if one considers a semimartingale in a smaller filtration to which it is adapted then it remains a semimartingale, although the decomposition may change. Recent developments in Mathematical Finance, and especially within the theory of credit risk, has led to the consideration of the projection of processes onto small filtrations to which they are no longer adapted. In this article we develop a general theory of how these projections behave.

To be more precise, martingales, supermartingales, and quasimartingales all retain their nature and are of course semimartingales in the smaller filtration. However the situation for local martingales is more delicate. Indeed, even in the classic case of having the local martingale still adapted, Stricker observed [30] that a local martingale need not remain a local martingale in a smaller filtration. It only gets worse when considering optional projections onto a filtration to which the local martingale is not adapted. We establish what is in fact true, and provide an explicit case study for projections of the inverse Bessel (3) process. Another illustrative example is inspired by recent results in credit risk theory.

\section{Filtration SHRinkage}

There has been much work on the expansion of filtrations (see for example Chap. 6 of [27]) but little has been done on the converse: the shrinkage of filtrations. Perhaps the best known result in this direction is Stricker's theorem:

Theorem 2.1 (Stricker's theorem). Let $X$ be a semimartingale for a filtration $\mathbb{G}$ and let $\mathbb{F}$ be a subfiltration of $\mathbb{G}$ such that $X$ is adapted to $\mathbb{F}$. Then $X$ remains a semimartingale for $\mathbb{F}$.

\footnotetext{
Keywords and phrases. Filtration shrinkage, filtration expansion, martingales, semimartingales, local time, Brownian motion.

${ }^{1}$ Humboldt Universität, Berlin, Germany.

2 Supported in part by NSF Grant DMS-0906995; Statistics, Columbia University, New York, NY 10027, USA.

protter@stat. columbia.edu
} 
One can see [30] or [23] for the original proof, or [27], p. 53 for a simpler argument. In Stricker's theorem, one assumes that $X$ is adapted to the smaller filtration; one may well ask if this is true for general semimartingales for a filtration even smaller than the natural filtration of $X$ itself. In this paper, we show this result en passant, but what we are really interested in, is what happens to local martingales when projected onto smaller filtrations. We begin with a very elementary and well known result, which uses, as most of our results do, the following caveat:

Caveat 1. If not stated otherwise, all filtrations in this paper will be assumed to satisfy the usual hypotheses ${ }^{1}$. Note that this assumption will not be made in our discussion of local martingales viewed as measures on the predictable $\sigma$-field.

Caveat 2. All processes $X=\left(X_{t}\right)_{t \geq 0}$ under consideration for filtration shrinkage will be implicitly assumed to possess the property that $X_{t} \in L^{1}$ for all $t \geq 0$.

From now on we fix a filtration $\mathbb{G}$ on a given probability space $(\Omega, \mathcal{G}, P)$ and a subfiltration $\mathbb{F}$ of $\mathbb{G}$.

Theorem 2.2. Let $X$ be a martingale for the filtration $\mathbb{G}$. Then the optional projection of $X$ onto $\mathbb{F}$ is again a martingale, for the filtration $\mathbb{F}$.

Proof. First let us observe that on a time interval $[0, n]$ we have that $\left(X_{t}\right)_{0 \leq t \leq n}$ is a uniformly integrable martingale of "Class (D)". Therefore for any $\mathbb{F}$ stopping time $\tau$ bounded by $n$ we have that the optional projection ${ }^{\circ} X_{\tau}=E\left\{X_{\tau} \mid \mathcal{F}_{\tau}\right\}$, and hence the optional projection is well defined on $[0, t]$ for any $t \leq n$. Since $n$ is arbitrary, it is well defined on $[0, \infty)$. Since the optional projection of $X$ onto $\mathbb{F}$, denoted ${ }^{o} X$, at time $t$ is a.s. equal to $E\left(X_{t} \mid \mathcal{F}_{t}\right)$, we have that

$$
\begin{aligned}
E\left({ }^{o} X_{t} \mid \mathcal{F}_{s}\right) & =E\left(E\left(X_{t} \mid \mathcal{F}_{t}\right) \mid \mathcal{F}_{s}\right)=E\left(X_{t} \mid \mathcal{F}_{s}\right) \\
& =E\left(E\left(X_{t} \mid \mathcal{G}_{s}\right) \mid \mathcal{F}_{s}\right)=E\left(X_{s} \mid \mathcal{F}_{s}\right) \\
& ={ }^{o} X_{s} .
\end{aligned}
$$

Theorem 2.3. Let $X \geq 0$ be a supermartingale for $\mathbb{G}$. Then ${ }^{\circ} X$ is a supermartingale for $\mathbb{F}$.

Proof. For the optional projection for supermartingales, we need to be a little more careful than for martingales. However since we assume here that $X$ is nonnegative, there is no problem (see for example [6], paragraph 43, p. 115). After this remark, the proof is identical to that of Theorem 2.2, with equality replaced by less than or equal.

Our goal is to find general conditions on a local martingale for $\mathbb{G}$ which ensure that it is also a local martingale for $\mathbb{F}$. We first show the result for a subclass of semimartingales known as quasimartingales, which is of interest in its own right. Theorem 2.4 extends Stricker's theorem (Thm. 2.1), and is due to Brémaud and Yor [2]. Theorem 3.5 in turn extends Theorem 2.4 to the more general case of semimartingales. We need to recall a standard definition:

Definition 2.1. A stochastic process $X$ is a quasimartingale for $\mathbb{G}$ if

$$
\operatorname{Var}(X, \mathbb{G})=\sup _{\tau} \operatorname{Var}_{\tau}(X, \mathbb{G})<\infty
$$

where

$$
\operatorname{Var}_{\tau}(X, \mathbb{G})=E\left(\sum_{i=0}^{n}\left|E\left\{X_{t_{i}}-X_{t_{i+1}} \mid \mathcal{G}_{t_{i}}\right\}\right|\right)
$$

for a finite partition $\tau=\left\{t_{0}, t_{1}, \ldots, t_{n}\right\}$.

\footnotetext{
${ }^{1}$ See [27], p. 3 for a definition of the usual hypotheses.
} 
Theorem 2.4. If $X$ is a quasimartingale for $\mathbb{G}$ then ${ }^{\circ} X$ is a quasimartingale for $\mathbb{F}$, where ${ }^{o} X$ denotes the optional projection of $X$ onto $\mathbb{F}$.

Proof. We know by Rao's theorem that a quasimartingale is a difference of two submartingales [27], p. 118, hence the theorem follows from Theorem 2.3.

\section{LOCAL MARTINGALES}

The notion of a local martingale was first introduced by Itô and Watanabe in [10].

Definition 3.1. A local martingale for $\mathbb{G}$ is a càdlàg process $X$ adapted to the filtration $\mathbb{G}$ such that there exists a sequence of stopping times $\left(T_{n}\right)_{n \geq 1}$ in $\mathbb{G}$ increasing to $\infty$ a.s. such that for each $n, X_{t \wedge T_{n}} 1_{\left\{T_{n}>0\right\}}$ is a martingale for $\mathbb{G}$. A sequence $\left(T_{n}\right)_{n \geq 1}$ of such stopping times is called a reducing sequence or a localizing sequence.

We will dispense with the term $1_{\left\{T_{n}>0\right\}}$ throughout this paper, since we will assume all of our processes are non random at time $t=0$, hence the indicator term is not necessary.

We have the following two useful results concerning local martingales, due to Kazamaki [18].

Theorem 3.1 (Krickeberg decomposition for local martingales). Let $X$ be a local martingale. Then $\|X\|_{1} \equiv$ $\sup _{n} E\left(\left|X_{T_{n}}\right|\right)$, for every reducing sequence of stopping times $\left(T_{n}\right)$ with $T_{n}<\infty$ a.s. each $n$, and $\lim _{n \rightarrow \infty} T_{n}=\infty$. If $\|X\|_{1}<\infty$, then there exist two positive local martingales $X^{1}$ and $X^{2}$ such that

$$
X=X^{1}-X^{2} \quad \text { and } \quad\|X\|_{1}=\left\|X^{1}\right\|_{1}+\left\|X^{2}\right\|_{1},
$$

and this decomposition is unique.

Theorem 3.2. A local martingale $X$ is a quasimartingale if and only if $\|X\|_{1}<\infty$.

We can use Kazamaki's results to extend the result of Bŕemaud and Yor (Thm. 2.4) to semimartingales. Before we do that, however, we should clarify a detail concerning optional projections. We have the following result, which can be found in [6], p. 116. We state the result and also sketch its proof here, for the reader's convenience. Dellacherie and Meyer attribute the result to Chen Pei-De. Recall that optional projections exist for any nonnegative process.

Theorem 3.3. Let $X$ be a $\mathbb{G}$ measurable process, and suppose ${ }^{\circ}|X|$ is indistinguishable from a finite valued process, where the optional projection is taken on $\mathbb{F} \subset \mathbb{G}$. If we let $Y={ }^{o}\left(X^{+}\right)-^{o}\left(X^{-}\right)$, then $Y$ defines an optional projection of $X$.

Proof. It is useful to note that in order to verify the hypothesis that ${ }^{o}|X|$ s indistinguishable from a finite valued process, it suffices to use Meyer's section theorem and to check that for any stopping time $\tau$ we have that

$$
E\left(\left|X_{\tau}\right| 1_{\{\tau<\infty\}} \mid \mathcal{F}_{\tau}\right)<\infty \text { a.s. }
$$

This in turn is equivalent to verifying that $E\left(X \tau 1_{\{\tau<\infty\}} \mid \mathcal{F}_{\tau}\right)$ exists. But this implies that

$$
Y_{\tau} 1_{\{\tau<\infty\}}=E\left(X_{\tau} 1_{\{\tau<\infty\}} \mid \mathcal{F}_{\tau}\right) \text { a.s. }
$$

which is formally the definition of the optional projection. That $Y$ is uniquely so characterized follows from another application of Meyer's section theorem.

The next theorem shows that if the projection of a semimartingale onto a smaller filtration exists, then the projection is a semimartingale in the smaller filtration. The following lemma will be needed in the proof of the theorem (and again in the proof of Thm. 3.7), so we begin with it. 
Lemma 3.4. Let $X$ be a $\mathbb{G}$ measurable process, and suppose that the optional projection onto $\mathbb{F},{ }^{o} X$, exists. Let $T$ be a finite valued $\mathbb{F}$ stopping time. Then ${ }^{o}\left(X_{t \wedge T}\right)=\left({ }^{o} X\right)_{t \wedge T}$.

Proof. Let $S$ be a finite valued $\mathbb{F}$ stopping time, and let $X^{T}$ denote the stopped process $X_{t \wedge T}$, and also let $Y=X^{T}$. By replacing $S$ with $S \wedge T$ if necessary, we can assume without loss of generality that $S \leq T$. Then

$$
\begin{aligned}
{ }^{o} Y_{S} & =E\left(Y_{S} \mid \mathcal{F}_{S}\right)=E\left(X_{S}^{T} \mid \mathcal{F}_{S}\right)=E\left(X_{T \wedge S} \mid \mathcal{F}_{S}\right) \\
& =E\left(X_{T \wedge S}\left|\mathcal{F}_{T}\right| \mathcal{F}_{S}\right)=E\left(X_{T \wedge S} \mid \mathcal{F}_{t \wedge S}\right)
\end{aligned}
$$

by definition of the optional projection. On the other hand $\operatorname{consider}^{o}\left(X_{S}^{T}\right)$. We have

$$
{ }^{o}\left(X_{S}^{T}\right)={ }^{o}\left(X_{t \wedge S}\right)=E\left(X_{T \wedge S} \mid \mathcal{F}_{T \wedge S}\right)
$$

and since the two right sides of equations (3.1) and (3.2) are equal, the lemma is proved.

Theorem 3.5. Let $X$ be a semimartingale for $\mathbb{G}$ such that ${ }^{\circ}|X|$ exists and is a finite valued process, where ${ }^{o}|X|$ denotes the optional projection of $|X|$ onto the filtration $\mathbb{F} \subset \mathbb{G}$. Then ${ }^{\circ} X$ exists by Theorem 3.3, and it is an $\mathbb{F}$ semimartingale.

Proof. Since $X$ is a $\mathbb{G}$ semimartingale, let $X=M+A$ be a decomposition, with $M$ a local martingale and $A$ a process with paths of finite variation. For the local martingale $M$ we let $\left(\tau_{n}\right)_{n=1,2, \ldots}$ be a reducing sequence of stopping times, so that $\left(M_{t \wedge \tau_{n}}\right)_{t \geq 0}$ is a uniformly integrable martingale for each $n \geq 1$. Let us fix an $n$, and let $M_{t}^{n} \equiv M_{t \wedge \tau_{n}}$. Note that we have $E\left(\left|M_{\infty}^{n}\right|\right)=E\left(\left|X_{t \wedge \tau_{n}}\right|\right)<\infty$, and by Jensen's inequality we have $\left\|M^{n}\right\|_{1}<\infty$. Therefore by Krickeberg's decomposition we have two uniquely determined positive martingales $M^{1, n}, M^{2, n}$ such that $M^{n}=M^{1, n}-M^{2, n}$ and $\left\|M^{n}\right\|_{1}=\left\|M^{1, n}\right\|_{1}+\left\|M^{2, n}\right\|_{1}$. We now take the optional projection of each, and we have

$$
{ }^{o} M^{n}={ }^{o} M^{1, n}-{ }^{o} M^{2, n} \text { on }\left[0, \tau_{n}\right] .
$$

Since the Krickeberg decomposition is unique, and the optional projection is also uniquely defined, we can combine equation (3.3) using that

$$
{ }^{o} M_{t \wedge \tau_{n}}^{n+1}={ }^{o} M_{t \wedge \tau_{n}}^{n}
$$

by Lemma 3.4 to define ${ }^{o} M$ on $[0, \infty)$. By Theorem 3.8 we know that ${ }^{o} M^{i, n}$ is a nonnegative supermartingale for $i=1,2$. Therefore ${ }^{o} M$ is the difference of two supermartingales, whence a semimartingale. The finite variation term $A$ is easier: for $A \mathbb{G}$ optional it is a classical result due to Dellacherie that we can decompose $A$ into increasing, nonnegative optional processes such that $A_{t}=A^{-}-A^{-}$, and moreover $|d A|_{t}=A_{t}^{+}+A_{t}^{-}$. (It is of course the fact that $A^{+}$and $A^{-}$can be taken optionally measurable that is due to Dellacherie; it is due to Lebesgue that we can do this path by path for each fixed $\omega$.) Therefore since ${ }^{o}|X|$ exists and is finite by hypothesis which implies ${ }^{o} X$ is well defined by Theorem 3.3, and we have seen that ${ }^{\circ} M$ exists and is finite valued, by linearity we have that

$$
{ }^{o} A={ }^{o} X-{ }^{o} M
$$

exists as well, and is uniquely defined. Since $A^{+}$is an increasing process, so also is ${ }^{o} A^{+}$, and analogously for $A^{-}$and ${ }^{o} A^{-}$, hence ${ }^{o} A={ }^{o} A^{+}-{ }^{o} A^{-}$is a finite variation process, and also a semimartingale. Therefore ${ }^{o} X$ is a semimartingale.

Among many other results, Stricker [30] showed the following:

Theorem 3.6. Let $X$ be a positive local martingale for $\mathbb{G}$, and assume that $X$ is adapted to the subfiltration $\mathbb{F}$. Then $X$ then $X$ is also a local martingale for $\mathbb{F}$.

Corollary 3.1. If $X$ is a local martingale for $\mathbb{G}$ with $\|X\|_{1}<\infty$, and if $X$ is adapted to $\mathbb{F}$, then $X$ is also a local martingale for $\mathbb{F}$.

Proof. Since $\|X\|_{1}<\infty$ we know that $X$ is a quasimartingale, hence a fortiori a special semimartingale, and the result follows from Theorem 3.6. 
It is interesting to note that when a local martingale is no longer assumed to be positive, Stricker gives an example to show that even in the case where $X$ is adapted to the smaller filtration, one nevertheless needs the extra assumption that $X$ is a special supermartingale in the smaller filtration to ensure that $X$ remains a local martingale in the smaller filtration.

When $X$ is not adapted to the smaller filtration $\mathbb{F}$, the situation is more complicated, as our case study for the inverse Bessel process will show.

The next theorem is our main result in this section:

Theorem 3.7. Let $X$ be a local martingale for the filtration $\mathbb{G}$, and let ${ }^{\circ} X$ denote the optional projection of $X$ onto the subfiltration $\mathbb{F}$. ${ }^{o} X$ is a local martingale for $\mathbb{F}$ if there exists a sequence of reducing stopping times $\left(T_{n}\right)_{n \geq 1}$ for $X$ in $\mathbb{G}$ which are also stopping times in $\mathbb{F}$. Conversely, if $X$ is positive and ${ }^{\circ} X$ is a local martingale for $\mathbb{F}$, then a reducing sequence of stopping times for ${ }^{\circ} X$ in $\mathbb{F}$ is also a reducing sequence for $X$ in $\mathbb{G}$.

Proof. Since $M_{t}^{n}=X_{t \wedge T_{n}}$ is a martingale for $\mathbb{G}$, the optional projection of $M^{n}$ onto $\mathbb{F}$ is also a martingale for $\mathbb{F}$. Since $T_{n}$ is a stopping time in $\mathbb{F}$, so also is $t \wedge T_{n}$. Using Lemma 3.4, we have ${ }^{o} X_{t \wedge T_{n}}={ }^{o} M_{t}^{n}$, and therefore ${ }^{o} X_{t \wedge T_{n}}$ is a martingale for $\mathbb{F}$, for each $n$, and we have that ${ }^{\circ} X$ is a local martingale for $\mathbb{F}$ with reducing sequence $\left(T_{n}\right)$.

For the converse, suppose ${ }^{o} X$ is a positive local martingale for $\mathbb{F}$. Let $\left(\tau_{n}\right)_{n>1}$ be a reducing sequence. Then $\left(\tau_{n}\right)_{n \geq 1}$ are also stopping times for the filtration $\mathbb{G}$, and if $Y_{t}={ }^{o} X_{t \wedge \tau_{n}}$, then $Y$ is a positive martingale, hence its expectation is 1 . But $E\left(X_{t \wedge \tau_{n}}\right)=E\left({ }^{o} X_{t \wedge \tau_{n}}\right)=E\left(Y_{t}\right)=E\left(X_{0}\right)$ is constant, and this coupled with the hypothesis that $X$ is positive, gives that $X_{t \wedge \tau_{n}}$ is a martingale for $\mathbb{G}$, because a positive local martingale with constant expectation is a true martingale.

Corollary 3.2. Let $X$ be a local martingale for the filtration $\mathbb{G}$ and let $\mathbb{E} \subset \mathbb{F}$ be a subfiltration of $\mathbb{F} \subset \mathbb{G}$. If both $X$ and the optional projection of $X$ onto $\mathbb{E}$ are local martingales, then the projection of $X$ onto $\mathbb{F}$ is also a local martingale.

Recall the fairly standard notation that for a process $X, \Delta X_{s}=X_{s}-X_{s-}$, the jump of $X$ at time $s$.

Theorem 3.8. Let $X>0$ be a local martingale relative to $(P, \mathbb{G})$. Let ${ }^{\circ} X$ be its optional projection onto the subfiltration $\mathbb{F}$. Then ${ }^{\circ} X$ is a supermartingale. Assume that it is special, with canonical decomposition ${ }^{o} X_{t}=M_{t}-A_{t}$, that $\langle M, M\rangle$ exists, and that $d A_{t} \ll \mathrm{d}\langle M, M\rangle_{t}$ with predictable density process $\left(c_{s}\right)$. Assume that $c_{s} \Delta M_{s}>-1$. Denote by $Z$ the unique solution of

$$
Z_{t}=1+\int_{0}^{t} Z_{s-} c_{s} \mathrm{~d} M_{s} .
$$

Since $c_{s} \Delta M_{s}>-1$ we know that $Z$ is a strictly positive process. Assume in addition that $Z$ is in fact a martingale, and that the probability measure $Q$, defined consistently by the density $Z_{t}$ on $\mathcal{G}_{t}$ for each $t \geq 0$, admits an extension to $\mathcal{G}$. Then ${ }^{\circ} X$ is a local martingale with respect to $Q$ in the smaller filtration $\mathbb{F}$.

Proof. By the predictable version of the Meyer-Girsanov theorem (cf. [27], p. 135) we have that under $Q$ the process

$$
\begin{aligned}
M_{t}-\int_{0}^{t} \frac{1}{Z_{s-}} \mathrm{d}\langle Z, M\rangle_{s} & =M_{t}-\int_{0}^{t} \frac{1}{Z_{s-}} Z_{s-} c_{s} \mathrm{~d}\langle M, M\rangle_{s} \\
& =M_{t}-\int_{0}^{t} c_{s} \mathrm{~d}\langle M, M\rangle_{s} \\
& =M_{t}-A_{t}
\end{aligned}
$$

is a local martingale in the filtration $\mathbb{F}$. 
Example 3.1. Suppose that, in the context of the preceding theorem, we have

$$
\left|E\left(X_{t}-X_{s} \mid \mathcal{G}_{s}\right)\right| \leq K(t-s)
$$

for any $s<t$. Then we also have

$$
\begin{aligned}
E\left({ }^{o} X_{t}-{ }^{o} X_{s} \mid \mathcal{F}_{s}\right) & =E\left(E\left(X_{t} \mid \mathcal{F}_{t}\right)-E\left(X_{s} \mid \mathcal{F}_{s}\right) \mid \mathcal{F}_{s}\right) \\
& =E\left(X_{t}-X_{s} \mid \mathcal{F}_{s}\right) \\
& =E\left(E\left(X_{t}-X_{s} \mid \mathcal{G}_{s}\right) \mid \mathcal{F}_{s}\right) \\
& \leq K(t-s) .
\end{aligned}
$$

It follows fairly easily that ${ }^{o} X$ is special with a canonical decomposition ${ }^{o} X_{t}=M_{t}-A_{t}$, and that the paths $t \mapsto A_{t}$ are continuous a.s. Therefore $\left[{ }^{o} X,{ }^{o} X\right]=[M, M]$, and it follows that $\left\langle{ }^{o} X,{ }^{o} X\right\rangle=\langle M, M\rangle$. We can in principle find $A$ from ${ }^{\circ} X$ itself via the Laplacian approach: define

$$
A_{t}^{h}=\frac{1}{h} \int_{0}^{t}\left({ }^{o} X_{s}-E\left({ }^{o} X_{s+h} \mid \mathcal{F}_{s}\right) \mathrm{d} s=\frac{1}{h} \int_{0}^{t}\left({ }^{o} X_{s}-E\left(X_{s+h} \mid \mathcal{F}_{s}\right) \mathrm{d} s\right.\right.
$$

and then we know that $\lim _{h \rightarrow 0} A_{T}^{h}=A_{T}$ with convergence in $L^{1}$, whenever ${ }^{o} X$ is in Class D. (See, e.g., [27], p. 150.) Our Lipschitz hypothesis implies that the paths of $A$ are absolutely continuous a.s., as well. If we further assume $\mathrm{d}\left\langle{ }^{\circ} X,{ }^{o} X\right\rangle_{t} \ll \mathrm{d} t$, then we can write

$$
A_{t}=\int_{0}^{t} h_{s} \mathrm{~d} s \text { and } \quad\left\langle{ }^{o} X,{ }^{o} X\right\rangle_{t}=\int_{0}^{t} j_{s} \mathrm{~d} s
$$

and then

$$
c_{s}=\frac{\mathrm{d} A_{s}}{\mathrm{~d}\left\langle{ }^{o} X,{ }^{o} X\right\rangle_{s}}=\frac{h_{s}}{j_{s}} 1_{\left\{j_{s} \neq 0\right\}} .
$$

Therefore letting $Z$ solve $Z_{t}=1+\int_{0}^{t} Z_{s-} c_{s} \mathrm{~d}\left({ }^{o} X_{s}+A_{s}\right)$ and assuming that $Z$ is in fact a positive martingale, we obtain for each finite horizon $T$ an equivalent risk neutral measure for $\left({ }^{\circ} X, \mathbb{F}\right)$, in the sense of No Free Lunch with Vanishing Risk; cf. Delbaen and Schachermayer [5]. This sort of approach is useful in the modelling of credit risk (see, e.g., [3], [13] and [29]).

One may ask what happens in the case of discrete time. This issue is moot in the following sense: Meyer showed in 1972 ([22]) that all discrete time local martingales are in fact martingales, up to the usual integrability assumption. More precisely, he proved the following result:

Theorem 3.9. Let $\left(X_{n}\right)_{n \in \mathbb{N}}$ be a process adapted to an underlying filtration $\left(\mathcal{F}_{n}\right)_{n \in \mathbb{N}}$ such that $X_{0} \in L^{1}$. Then the following are equivalent:

(1) $X$ is a local martingale;

(2) For each $n$ one has $E\left(\left|X_{n+1}\right| \mid \mathcal{F}_{n}\right)<\infty$ a.s., and $E\left(X_{n+1} \mid \mathcal{F}_{n}\right)=X_{n}$.

\section{Supermartingales as measures on product Space}

In this section we show how the preceding results on optional projections can be derived from the identification of quasi- and supermartingales as measures on the product space

$$
\bar{\Omega}=\Omega \times(0, \infty]
$$

endowed with the predictable $\sigma$-field; $c f$. [7], [8], and [21]. 
Let $\mathcal{P}_{\mathbb{G}}$ and $\mathcal{P}_{\mathbb{F}}$ denote the predictable $\sigma$-fields on $\bar{\Omega}$ which are associated to the right-continuous filtrations $\mathbb{G}$ and $\mathbb{F} \subset \mathbb{G}$, respectively. In this section we do not assume that the filtrations are completed, because we are going to introduce new measures. The second component $\zeta(\omega, t):=t$ will be viewed as a lifetime.

Under some regularity conditions on $\mathbb{G}$ of topological type (see [8]), an adapted right-continuous process $X$ is a quasimartingale for $\mathbb{G}$ if and only if there is a unique signed measure $P^{X}$ with finite total variation on the predictable $\sigma$-field $\mathcal{P}_{\mathbb{G}}$ such that

$$
P^{X}\left(A_{t} \times(t, \infty]\right)=E\left(X_{t} ; A_{t}\right)
$$

for any $t \geq 0$ and $A_{t} \in \mathcal{G}_{t}$. $X$ is a nonnegative supermartingale iff the measure $P^{X}$ is nonnegative, it is a martingale iff $P^{X}(\zeta<\infty)=0$, and it is a potential iff $P^{X}(\zeta=\infty)=0$. Moreover, a nonnegative supermartingale $X$ is a local martingale if and only if there is an increasing sequence of stopping times $\left(T_{n}\right)$ in $\mathbb{G}$ which predicts the lifetime $\zeta$. For $X_{0}=1$ this means that $P^{X}$ is a probability measure on $\mathcal{P}_{\mathbb{G}}$ such that

$$
P^{X}\left(T_{n}<\zeta \text { for any } n, \zeta=\lim _{n} T_{n}\right)=1 .
$$

Clearly, the restriction of $P^{X}$ to the $\sigma$-field $\mathcal{P}_{\mathbb{F}} \subset \mathcal{P}_{\mathbb{G}}$ takes the form

for any $t \geq 0$ and $A_{t} \in \mathcal{F}_{t}$, and so we have

$$
P^{X}\left(A_{t} \times(t, \infty]\right)=E\left({ }^{o} X_{t} ; A_{t}\right)
$$

$$
\left.P^{X}\right|_{\mathcal{P}_{\mathbb{F}}}=P^{o} X
$$

This yields alternative proofs of Theorems 2,3 , and 4 , since the properties of being concentrated on $\zeta=\infty$, of having finite total variation, and of being positive are obviously preserved if the measure $P^{X}$ is restricted to the smaller $\sigma$-field $\mathcal{P}_{\mathbb{F}}$. Theorem 8 follows in a similar manner: if the lifetime $\zeta$ is predicted by a sequence of stopping times $\left(T_{n}\right)$ in the subfiltration $\mathbb{F}$ then property (4.2) can be read as a property of the restriction of $P^{X}$ to the smaller $\sigma$-field $\mathcal{P}_{\mathbb{F}}$, i.e., as a property of $P^{\circ} X$, and hence as the local martingale property of the optional projection ${ }^{\circ} X$.

\section{A CASE Study: The INVERSe Bessel PROCESS}

In this section we focus on the inverse Bessel (3) process, viewed as a functional of three-dimensional Brownian motion, and we study its optional projections onto the subfiltrations generated by one or two of the three Brownian components. The inverse Bessel process is in fact one of the first examples of a local martingale which appeared in the literature as soon as the notion of a local martingale had been introduced.

Let $\left(B_{t}\right)_{t \geq 0}=\left(B_{t}^{1}, B_{t}^{2}, B_{t}^{3}\right)_{t \geq 0}$ denote a standard three-dimensional Brownian motion starting at some point $x_{0} \neq 0$, say at $x_{0}=(1,0,0)$. Then $\left(\left\|B_{t}\right\|\right)_{t \geq 0}$ is a Bessel (3) process with initial value 1 . It is well known that the inverse Bessel process

is a local martingale which is localized by the stopping times

$$
M_{t}:=\left\|B_{t}\right\|^{-1}, \quad t \geq 0,
$$

$$
T_{n}=\inf \left\{t>0 \mid\left\|B_{t}\right\| \leq \frac{1}{n}\right\} .
$$

Note that $M$ is not a martingale since (as can be seen as consequence of equation (5.2))

$$
E\left(M_{t}\right)=2 \Phi\left(\frac{1}{\sqrt{t}}\right)-1
$$

converges to 0 as $t$ tends to $\infty$ a.s. and in $L^{1}$; here and in the sequel $\Phi$ denotes the distribution function of a $N(0,1)$ random variable. Thus $M$ is a potential, but the local martingale property shows that it is not a 
potential of class (D). Much is known about the inverse Bessel process, and one can consult (as one example among many) the recent article [25] or the classic text [28]. See also the earlier references [20] and [17] where the inverse Bessel process is used to illustrate the fact that a uniformly integrable process is not necessarily of class (D).

One way of checking the local martingale property is to apply Itô's formula, first to $U:=\|B\|$ and then to $M=U^{-1}$. This yields $\mathrm{d} U=\mathrm{d} W+U^{-1} \mathrm{~d} t$ and

$$
\mathrm{d} M=-M^{2} \mathrm{~d} W
$$

where $\mathrm{d} W:=M(B, \mathrm{~d} B)$ defines a one-dimensional Wiener process, due to Lévy's characterization of the Wiener process as a continuous local martingale with quadratic variation $\langle W, W\rangle=t$. Each stopped process $M_{T_{n} \wedge t}$, $t \geq 0$, is a martingale since it is bounded by $n$, and the stopping times $T_{n}$ converge to $\infty$ since any point is polar for Brownian motion in $R^{3}$. Thus we have verified that $M$ is indeed a local martingale. Alternatively, we can see that the inverse Bessel process is a strict local martingale as a consequence of a result of Mijatović and Urusov [24] or alternatively of Blei and Engelbert [1]. Indeed it follows from their results that the solution of $\mathrm{d} M=\sigma(M) \mathrm{d} W$ is a strict local martingale if $\int_{c}^{\infty} x \sigma^{-2}(x) \mathrm{d} x<\infty$ for some $c>0$. Since in the case of the inverse Bessel process $\sigma(x)=-x^{2}$, it meets this criterion and hence is a (strict) local martingale.

Instead of applying stochastic calculus, we can also argue in terms of probabilistic potential theory, as it was done in [21]. To this end, note that the three-dimensional Green function $h(x)=\|x\|^{-1}$ satisfies

$$
\Delta h=-4 \pi \delta_{0}
$$

(in the distributional sense). In particular, $h$ is harmonic on $R^{3} \backslash\{0\}$. Thus the process $M=h(B)$ obtained by observing the function $h$ along the paths of three-dimensional Brownian motion is a bounded martingale up to each stopping time $T_{n}$, and so it is indeed a local martingale.

Remark 5.1. In view of equation (4.1), with $X=M=h(B)$, the measure $P^{M}$ associated to the local martingale $M$ can be identified with Doob's $h$-path process $P^{h}$, i.e.,

$$
P^{M}\left(A_{t} \times(t, \infty]\right)=E\left(h\left(X_{t}\right) ; A_{t}\right)=E^{h}\left(A_{t} \cap\{\zeta>t\}\right)
$$

for $t \geq 0$ and $A_{t} \in \mathcal{G}_{t}$. Under $P^{h}$ the life time $\zeta$ is finite a.s., since $M$ is a potential. More precisely, $P^{h}$ can be viewed as a conditioning of the original Brownian motion $B$ to go to the origin in finite time, driven by the stochastic differential equation

$$
\mathrm{d} B=\mathrm{d} B^{h}-\nabla \log h(B) \mathrm{d} t=\mathrm{d} B^{h}-\frac{B}{\|B\|^{2}} \mathrm{~d} t,
$$

where $B^{h}$ is a three-dimensional Brownian motion under $P^{h}$.

We are now going to look at the optional projection $N$ of the local martingale $M$ onto the smaller filtrations $\mathbb{F}$ generated, respectively, by the two-dimensional Brownian motion $\left(B^{1}, B^{2}\right)$ and the one-dimensional Brownian motion $B^{1}$. Our aim is to check whether the supermartingale $N$ inherits the local martingale property or not. To this end, we compute in both cases the Doob-Meyer decomposition of $N$, and we also clarify the structure of the corresponding measure $P^{N}$ on the predictable $\sigma$-field $\mathcal{P}_{\mathbb{F}}$.

Note first that, in view of equation (5.1), the local martingale property is clearly lost if we take the trivial filtration where the optional projection simply consists in taking the expectations $N_{t}=E\left(M_{t}\right)$. In this case, the measure $P^{N}$ is simply the probability measure on the positive half-line with distribution function $F(t)=$ $1-E\left(M_{t}\right)$.

Let us now consider the case $\mathbb{F}=\sigma\left(B^{1}\right)$ where the subfiltration $\mathbb{F}$ is generated by the one-dimensional Brownian motion $B^{1}$. 
Theorem 5.1. The optional projection $N$ of the inverse Bessel process $M$ onto the filtration $\mathbb{F}=\sigma\left(B^{1}\right)$ is a supermartingale, but it is not a local martingale. More precisely:

i) The process $N$ is a supermartingale of the form $N_{t}=u\left(B_{t}^{1}, t\right)$, where

$$
u(x, t)=\sqrt{\frac{2 \pi}{t}} \exp \left(\frac{x^{2}}{2 t}\right)\left(1-\Phi\left(\frac{|x|}{\sqrt{t}}\right)\right)
$$

for $t>0$ and $u(x, 0)=x^{-1}$. The function $u$ is space-time superharmonic on $R \times[0, \infty)$ and satisfies

$$
\left(\frac{1}{2} \frac{\partial^{2}}{\partial x^{2}}+\frac{\partial}{\partial t}\right) u=-\frac{1}{t} \delta_{0}
$$

on $R \times(0, \infty)$ (in the distributional sense).

ii) $N$ is a potential of class $(D)$ of the form

$$
\begin{aligned}
N_{t} & =1+\int_{0}^{t} u_{x}\left(B_{s}^{1}, s\right) \mathrm{d} B_{s}^{1}-\int_{0}^{t} \frac{1}{s} \mathrm{~d} L_{s}^{0} \\
& =E\left(\int_{t}^{\infty} \frac{1}{s} \mathrm{~d} L_{s}^{0} \mid \mathcal{F}_{t}\right),
\end{aligned}
$$

where $L^{0}$ denotes the local time of $B^{1}$ at 0 . In particular, $N$ is a martingale up to the time that $B^{1}$ hits 0 , but not a local martingale.

Proof. Since $M$ is a nonnegative local martingale in the filtration $\mathbb{G}$, it is a fortiori a supermartingale. Therefore its projection onto the shrunken filtration $\mathbb{F}$ is a nonnegative supermartingale by Theorem 2.3. Let us compute its Doob-Meyer decomposition $N=Y-A$ where $Y$ is a local martingale and $A$ is a predictable increasing process such that $A_{0}=0$. To this end, we observe that

$$
\begin{aligned}
N_{t} & =E\left(M_{t} \mid \mathcal{F}_{t}\right) \\
& =E^{(2,3)}\left\{\left(\left(B_{t}^{1}\right)^{2}+\left(B_{t}^{2}\right)^{2}+\left(B_{t}^{3}\right)^{2}\right)^{-\frac{1}{2}}\right\} \\
& =u\left(B_{t}^{1}, t\right)
\end{aligned}
$$

where $E^{(2,3)}$ denotes expectation with respect to the second and third coordinates; the second line is justified by the independence of $B^{1}$ with $\left(B^{2}, B^{3}\right)$. The function $u$ is clearly given by

$$
u(x, t)=\int_{0}^{\infty}\left(x^{2}+t r^{2}\right)^{-\frac{1}{2}} r \mathrm{e}^{-r^{2} / 2} \mathrm{~d} r .
$$

Using the change of variables $y^{2}=x^{2} / t+r^{2}$ we obtain its explicit form (Eq. 5.2).

It is now straightforward to check that $u$ is a solution of the PDE (Eq. 5.3). Applying a time-dependent version of the Itô-Tanaka formula (see, e.g., [9]), we obtain equation (5.4). Thus $N$ admits the Doob-Meyer decomposition $N=Y-A$ with

$$
Y_{t}=1+\int_{0}^{t} u_{x}\left(B_{s}^{1}, s\right) \mathrm{d} B_{s}^{1}, \quad A_{t}=\int_{0}^{t} \frac{1}{s} \mathrm{~d} L_{s}^{0}
$$

Note that $A_{\infty}$ is integrable because $E\left(A_{t}\right) \leq E\left(Y_{t}\right) \leq Y_{0}=1$. Since $M_{t}$ converges to 0 in $L^{1}$ as $t$ tends to $\infty$, the same is true for $N$, and this implies

$$
\lim _{t \rightarrow \infty} Y_{t}=A_{\infty}=\int_{0}^{\infty} \frac{1}{s} \mathrm{~d} L_{s}^{0} \quad \text { in } L^{1} .
$$


In contrast to the local martingale $M$ which is not of class $(\mathrm{D})$, the process $N=u\left(B^{1}, \cdot\right)$ remains bounded for $t \geq t_{0}$ in view of equation (5.2). This allows us to conclude that the local martingale $Y$ is in fact of class (D), and that it is given by

$$
Y_{t}=E\left(\int_{0}^{\infty} \frac{1}{s} \mathrm{~d} L_{s}^{0} \mid \mathcal{F}_{t}\right)
$$

This implies the second formula in part ii), and so we have identified $N$ as the potential of class (D) generated by the increasing process $A$.

Remark 5.2. Note that the increasing process in the Doob-Meyer decomposition of $N$ has paths which are singular with respect to Lebesgue measure, while the local martingale term has a quadratic variation process which is absolutely continuous. Thus from a Mathematical Finance perspective, the inverse Bessel process $M$ does not yield arbitrage, but its projection $N$ onto the smaller filtration $\mathbb{F}=\sigma\left(B^{1}\right)$ does in fact yield arbitrage opportunities. See for example [26], pp. 180-183 for a fuller explanation of why this affects the absence or presence of arbitrage opportunities.

Remark 5.3. In view of equations (4.3) and (5.4) the measure $P^{N}$ is given by

$$
P^{N}\left(A_{t} \times(t, \infty]\right)=E\left(N_{t} ; A_{t}\right)=E\left(\int_{t}^{\infty} \frac{1}{s} \mathrm{~d} L_{s}^{0} ; A_{t}\right)
$$

for any $t \geq 0$ and $A_{t} \in \mathcal{F}_{t}$. Thus $P^{N}$ has a natural extension

$$
P^{N}=P \otimes \frac{1}{t} \mathrm{~d} L_{t}^{0}
$$

from the predictable $\sigma$-field $\mathcal{P}_{\mathbb{F}}$ to the product $\sigma$-field on $\Omega \times(0, \infty]$. This description of $P^{N}$ admits the following interpretation: observing only the first coordinate of Doob's conditional Brownian motion $P^{h}=P^{M}$ introduced in Remark 5.1, we see a one-dimensional Brownian motion with time-dependent drift $\partial \log u$ which is killed at the rate $\frac{1}{t} \mathrm{~d} L_{t}^{0}$.

We have seen that the local martingale property is lost if we project the inverse Bessel process $M$ onto the subfiltration generated by only one of the three Brownian components. The situation changes if we consider the larger subfiltration $\mathbb{F}=\sigma\left(B^{1}, B^{2}\right)$ generated by two of the components: since the point $(0,0)$ is polar for twodimensional Brownian motion, we do no longer have a local time appearing in the Doob-Meyer decomposition.

Theorem 5.2. The optional projection $N$ of the inverse Bessel process $M$ onto the filtration $\mathbb{F}=\sigma\left(B^{1}, B^{2}\right)$ is a again a local martingale. More precisely:

i) The process $N$ is a supermartingale of the form

$$
N_{t}=u\left(B_{t}^{1}, B_{t}^{2}, t\right)
$$

with

$$
u(x, y, t)=\frac{1}{\sqrt{2 \pi t}} \exp \left(\frac{x^{2}+y^{2}}{4 t}\right) K_{0}\left(\frac{x^{2}+y^{2}}{4 t}\right),
$$

where $K_{0}$ denotes the modified Bessel function of the second kind. The function $u$ is space-time superharmonic on $R^{2} \times[0, \infty)$ and space-time harmonic on $\left(R^{2}-\{(0,0)\}\right) \times(0, \infty)$.

ii) $N$ is is a local martingale which is localized by the increasing sequence of stopping times

$$
T_{n}=\inf \left\{t>0 \mid\left(B_{t}^{1}\right)^{2}+\left(B_{t}^{2}\right)^{2} \leq \frac{1}{n}\right\}
$$

with $T_{n} \nearrow \infty P$-a.s. 
Proof. It follows as in the preceding proof that $N$ is given by equation (5.6) with

$$
u(x, y, t)=\int_{-\infty}^{\infty}\left(x^{2}+y^{2}+t z^{2}\right)^{-\frac{1}{2}} \frac{1}{\sqrt{2 \pi}} e^{-\frac{z^{2}}{2}} \mathrm{~d} z
$$

This takes the explicit form described by equation (5.7). Since $K_{0}$ is a solution of the Bessel equation

$$
K_{n}^{\prime \prime}(z) z^{2}+K_{n}^{\prime}(z) z+K_{n}(z)\left(z^{2}-n^{2}\right)=0
$$

for $n=0$, it is easy to check the stated properties of $u$. Since $u$ is space-time harmonic outside of the origin, and since any point is polar for two-dimensional Brownian motion, it follows that $N$ is a local martingale which is localized by the sequence in $\left(T_{n}\right)$ in equation (5.8).

Alternatively, we can check the local martingale property directly by applying Theorem 3.7 , since the sequence $\left(T_{n}\right)$ clearly localizes the original local martingale $M$. This yields, without any explicit computation via equation (5.7), space-time harmonicity of the function $u$ in equation (5.9) outside of the origin.

Remark 5.4. The measure $P^{N}$ describes a two-dimensional Brownian motion $W=\left(B^{1}, B^{2}\right)$ conditioned to go to the origin in finite time, driven by the stochastic differential equation

$$
\mathrm{d} W=\mathrm{d} W^{u}-\nabla \log u(W, t) \mathrm{d} t
$$

where $W^{u}$ is a two-dimensional Brownian motion under $P^{N}$.

We conclude our discussion of the Bessel (3) process with the following example of filtration shrinkage which is due to Jeulin and Yor [16].

Example 5.1. If $B$ is a standard one dimensional Brownian motion starting at 0, with its natural filtration $\mathbb{G}$, then for all $t \geq 0$ we define $S_{t}=\sup _{s \leq t} B_{s}$, and $U_{t}=2 S_{t}-B_{t}$. Pitman's theorem is that $U$ is a Bessel (3) process. If we define $\mathbb{F}$ to be the natural filtration of $W=\left(W_{t}\right)_{t \geq 0}$, where $W$ is the Brownian motion given by:

$$
W_{t}=U_{t}-\int_{0}^{t} \frac{1}{U_{s}} \mathrm{~d} s
$$

then in the filtration $\mathbb{G}, W$ is a semimartingale with decomposition $W=V+A$, where $V=W-A$ is a martingale, and $A_{t}=2 S_{t}-\int_{0}^{t} U_{s}^{-1} \mathrm{~d} s$. That is, we have the two semimartingale decompositions:

$$
\begin{aligned}
U_{t} & =V_{t}+\left(2 S_{t}-\int_{0}^{t} \frac{1}{U_{s}} \mathrm{~d} s\right) \text { in the filtration } \mathbb{G} \\
U_{t} & =W_{t}+\int_{0}^{t} \frac{1}{U_{s}} \mathrm{~d} s \text { in the filtration } \mathbb{F}
\end{aligned}
$$

Both $W$ and $V$ are Brownian motions in their respective filtrations. Note that $d S_{t} \perp \mathrm{d} t$ a.s.; that is the paths are singular. From the perspective of Mathematical Finance, $U$ admits arbitrage in the filtration $\mathbb{G}$, but does not admit arbitrage in the smaller filtration $\mathbb{F}$. 


\section{IMPLICATIONS FOR FINANCE}

It is instructive to give an example from economics as to how the issue of filtration shrinkage might arise "naturally". The idea of this example is taken from [12]. We do not try to force this example into the inverse Bessel format, since we give it only to yield the intuition involved. Imagine one observes (in filtration $\mathbb{F}$ ) a stock price of the form

$$
\mathrm{d} X_{t}=\sigma \mathrm{d} B_{t}+b\left(t, X_{t}\right) \mathrm{d} t .
$$

where $b$ is assumed to be bounded. Suppose a group of large investors decides to buy or sell the stock whenever it crosses the level $c$, so as to keep the price steady at $c$. This could happen for example if a company spins-off a subsidiary and supports its stock price at a given level $c$ by buying a fraction $\alpha$ of shares when it falls below the level $c$, and another fraction $\beta$ of investors, wishing to unload the spin-off, sells the stocks when it rises above the level $c$. For simplicity, assume $\alpha=\beta$.

Let $\left\{0=t<t_{1}<\cdots<t_{n}=T\right\}$ be a partition of the time interval [0,T], and suppose the stock is observed at these times. If $X_{t_{i}}<c$ and $X_{t_{i+1}}>c$, then a sale of proportion $\alpha$ occurs with an assumed price impact of $\alpha\left(X_{t_{i+1}}-c\right)=\alpha\left|X_{t_{i+1}}-c\right|$. If $X_{t_{i}}>c$ and $X_{t_{i+1}}<c$, then a purchase of proportion $\alpha$ occurs with an assumed price impact of $\alpha\left(c-X_{t_{i+1}}\right)=\alpha\left|X_{t_{i+1}}-c\right|$. The cumulative effect on the stock price $X$ by such a repurchase plan will be

$$
\sum_{i=1}^{n-1} \alpha\left|X_{t_{i+1}}-c\right| 1_{\left\{\operatorname{sign}\left(X_{t_{i+1}}-c\right) \neq \operatorname{sign}\left(X_{t_{i}}-c\right)\right\}} .
$$

Let $\alpha L_{t}^{c}$ denote the limit, assuming for the moment we know it exists:

$$
\alpha L_{t}^{c}=\lim _{\left\|\pi^{n}\right\| \rightarrow 0} \alpha \sum_{t_{i} \in \pi^{n}}\left|X_{t_{i+1}}-c\right| 1_{\left\{\operatorname{sign}\left(X_{t_{i+1}}-c\right) \neq \operatorname{sign}\left(X_{t_{i}}-c\right)\right\}} .
$$

The impact of this repurchase plan yields a "new" price process:

$$
\mathrm{d} Y_{t}=\sigma \mathrm{d} B_{t}+b\left(t, X_{t}\right) \mathrm{d} t+\alpha \mathrm{d} L_{t}^{c} .
$$

The next theorem gives us that the limit of equation (6.2) actually does exist:

Theorem 6.1. The process $\alpha L_{t}^{c}$ exists as a limit in u.c.p. (uniform convergence on compact time sets, in probability). Moreover, $t \rightarrow L_{t}^{c}$ is singular with respect to $\mathrm{d} t$, a.s., and is the local time of $X$ at the level c.

Proof. Since $b$ is assumed bounded, by Girsanov's theorem there exists $P^{*}$ equivalent to $P$ such that $X$ is a $P^{*}$ continuous local martingale; hence $X$ is Brownian motion by Lévy's theorem. Let us take $\sigma=1$, so that $X$ is standard Brownian motion. Then by [9], p. 160 we have the limit (6.2) exists in u.c.p., and $L^{c}$ is the local time of $X$ at level $c$.

Therefore the observer naturally thinks there is an arbitrage opportunity provided by this behavior of the group of large traders. But perhaps in the larger filtration $\mathbb{G}$, which the observer does not see, more is happening that renders the perceived large trader behavior to be simply the actions of traders who ultimately are price takers rather than effectively large traders whose actions significantly affect the price. So the observer correctly sees the local time using his limited vision in the $\mathbb{F}$, but reality shows that in the larger filtration $\mathbb{G}$, the perceived arbitrage opportunity is in fact illusory.

Let us mention a second example. An approach to the modelling of credit risk that is quite current is that of reduced form models. An attempt to relate reduced form models as simply projections of structural models onto a smaller filtration is outlined in [11], and carried out to some extent in [3] and more generally in [13]. In this approach, it is implicit that the risk neutral measure of the original underlying process is still used for the projected process. This is not a problem since in the papers mentioned one is dealing with martingales. 
But once the underlying is a strict local martingale, care must be taken, since if the projected process is not a local martingale then the measure is no longer a risk neutral measure (or "equivalent martingale measure" as they are often called). There still may exist a risk neutral measure, if one can use (for example) a procedure such as the one indicated in Theorem 3.8. Theorem 5.2 provides an example. However this is not true in general, as is shown in Theorem 5.1. If one is dealing with positive processes, as we are in our treatment of local martingales, then one need not consider the seemingly more general situation of sigma martingales (see, e.g., [27] for a definition and properties of sigma martingales), since a positive sigma martingale is a local martingale.

The issue of martingales (which behave nicely in filtration shrinkage) as opposed to local martingales (which we have seen here need not behave nicely) is related to the existence of financial bubbles (see any of $[4,14,15]$ or [19]), where if the price process is a strict local martingale it can indicate the presence of a bubble.

Acknowledgements. We wish to acknowledge useful discussions on this subject with M. Emery, J. Jacod, S. Pal, and D. Sezer. We extend them our thanks. We also wish to thank an anonymous referee for his or her close reading of a first version of this paper, which resulted in many useful suggestions that have improved the readability of this paper.

\section{REFERENCES}

[1] S. Blei and H.J. Engelbert, On Exponential Local Martingales Associated with Strong Markov Continuous Local Martingales. Stoch. Process. Appl. 119 (2009) 2859-2880.

[2] P. Brémaud and M. Yor, Changes of Filtration and Probability Measures. Z. Wahrscheinlichkeitstheorie verw. Gebiete 45 (1978) 269-295.

[3] U. Çetin, R. Jarrow, P. Protter and Y. Yildirim, Modeling credit risk with partial information. Ann. Appl. Probab. 14 (2004) $1167-1178$.

[4] A. Cox and D. Hobson, Local martingales, bubbles and option prices. Finance Stoch. 9 (2005) 477-492.

[5] F. Delbaen and W. Schachermayer, The Mathematics of Arbitrage. Springer-Verlag, Heidelberg (2006).

[6] C. Dellacherie and P.A. Meyer, Probabilités et Potentiel: Chapitres V à VIII, Théorie des Martingales. Hermann, Paris (1980).

[7] H. Föllmer, The exit measure of a supermartingale. Z. Wahrscheinlichkeitstheorie verw. Gebiete 21 (1972) 154-166.

[8] H. Föllmer, On the representation of semimartingales. Ann. Probab. 1 (1973) 580-589.

[9] H. Föllmer, P. Protter and A.S. Shiryaev, Quadratic covariation and an extension of Itô's formula. Bernoulli 1 (2005) 149-169.

[10] K. Itô and S. Watanabe, Transformation of Markov processes by multiplicative functionals. Ann. Inst. Fourier 15 (1965) $15-30$.

[11] R. Jarrow and P. Protter, Structural versus Reduced Form Models: A New Information Based Perspective. J. Invest. Manage. 2 (2004) 34-43.

[12] R. Jarrow and P. Protter, Large Traders, Hidden Arbitrage, and Complete Markets. J. Bank. Financ. 29 (2005) $2803-2810$.

[13] R. Jarrow, P. Protter and D. Sezer, Information Reduction via Level Crossings in a Credit Risk Model. Finance Stoch. 11 (2007) 195-212.

[14] R. Jarrow, P. Protter and K. Shimbo, Asset price bubbles in a complete market. Adv. Math. Finance, in Honor of Dilip B. Madan (2006) 105-130.

[15] R. Jarrow, P. Protter and K. Shimbo, Asset price bubbles in an incomplete market. Math. Finance 20 (2010) $145-185$.

[16] T. Jeulin and M. Yor, Inégalité de Hardy, semimartingales, et faux-amis, in Séminaire de Probabilités XIII. Lect. Notes Math. 721, Springer, Heidelberg, Berlin, New York (1979) 332-359.

[17] G. Johnson and L.L. Helms, Class (D) Supermartingales. Bull. Am. Math. Soc. 69 (1963) 59-62.

[18] N. Kazamaki, Krickeberg's decomposition for local martingales, in Séminaire de Probabilités VI. Lecture Notes in Math. 258, Springer, Heidelberg, Berlin, New York (1972) 101-103.

[19] M. Loewenstein and G.A. Willard, Rational equilibrium asset-pricing bubbles in continuous trading models. J. Econ. Theory $91(2000)$ 17-58.

[20] P.A. Meyer, Probability and Potentials. Blaisdell, Waltham (1966).

[21] P.A. Meyer, La mesure de H. Föllmer en théorie des surmartingales, in Séminaire de Probabilités VI. Lecture Notes in Math. 258, Springer, Heidelberg, Berlin, New York (1971) 118-129.

[22] P.A. Meyer, Martingales and stochastic integrals. Lect. Notes Math. 284, Springer, Heidelberg, Berlin, New York (1972).

[23] P.A. Meyer, Sur un théorème de C. Stricker, in Séminaire de Probabilités XI. Lecture Notes in Math. 581, Springer, Heidelberg, Berlin, New York (1977) 482-489.

[24] A. Mijatović and M. Urusov, The Martingale Property of Certain Local Martingales. arXiv:0905.3701 (2009) 
[25] S. Pal and P. Protter, Analysis of continuous strict local martingales via h-transforms. Stoch. Process. Appl. 120 (2010) $1424-1443$.

[26] P. Protter, A Partial Introduction to Financial Asset Pricing Theory. Stoch. Process. Appl. 91 (2001) 169-203.

[27] P. Protter, Stochastic Integration and Differential Equations, 2nd edition, Version 2.1. Springer-Verlag, Heidelberg (2005).

[28] D. Revuz and M. Yor, Continuous martingales and Brownian motion, 3rd edition. Springer, Berlin-Heidelberg (1999).

[29] D. Sezer, Filtration shrinkage by level crossings of a diffusion. Ann. Probab. 35 (2007) 739-757.

[30] C. Stricker, Quasimartingales, martingales locales, et filtrations naturelles. Z. Wahrscheinlichkeitstheorie verw. Gebiete 39 (1977) 55-63. 